\title{
Recent advances into vanadyl, vanadate and decavanadate interactions with actin
}

\author{
S. Ramos, ${ }^{a b}$ J. J. G. Moura ${ }^{a}$ and M. Aureliano ${ }^{* b}$ \\ Received 2nd August 2011, Accepted 26th September 2011 \\ DOI: $10.1039 / \mathrm{c} 1 \mathrm{mt00124h}$
}

\begin{abstract}
Although the number of papers about "vanadium" has doubled in the last decade, the studies about "vanadium and actin" are scarce. In the present review, the effects of vanadyl, vanadate and decavanadate on actin structure and function are compared. Decavanadate ${ }^{51} \mathrm{~V}$ NMR signals, at $-516 \mathrm{ppm}$, broadened and decreased in intensity upon actin titration, whereas no effects were observed for vanadate monomers, at $-560 \mathrm{ppm}$. Decavanadate is the only species inducing actin cysteine oxidation and vanadyl formation, both processes being prevented by the natural ligand of the protein, ATP. Vanadyl titration with monomeric actin (G-actin), analysed by EPR spectroscopy, reveals a $1: 1$ binding stoichiometry and a $K_{\mathrm{d}}$ of $7.5 \mu \mathrm{M}^{-1}$. Both decavanadate and vanadyl inhibited G-actin polymerization into actin filaments (F-actin), with a $\mathrm{IC}_{50}$ of 68 and $300 \mu \mathrm{M}$, respectively, as analysed by light scattering assays, whereas no effects were detected for vanadate up to $2 \mathrm{mM}$. However, only vanadyl (up to $200 \mu \mathrm{M}$ ) induces $100 \%$ of G-actin intrinsic fluorescence quenching, whereas decavanadate shows an opposite effect, which suggests the presence of vanadyl high affinity actin binding sites. Decavanadate increases (2.6-fold) the actin hydrophobic surface, evaluated using the ANSA probe, whereas vanadyl decreases it $(15 \%)$. Both vanadium species increased the $\varepsilon$-ATP exchange rate $\left(k=6.5 \times 10^{-3} \mathrm{~s}^{-1}\right.$ and $4.47 \times 10^{-3} \mathrm{~s}^{-1}$ for decavanadate and vanadyl, respectively). Finally, ${ }^{1} \mathrm{H}$ NMR spectra of G-actin treated with

$0.1 \mathrm{mM}$ decavanadate clearly indicate that major alterations occur in protein structure, which are much less visible in the presence of ATP, confirming the preventive effect of the nucleotide on the decavanadate interaction with the protein. Putting it all together, it is suggested that actin, which is involved in many cellular processes, might be a potential target not only for decavanadate but above all for vanadyl. By affecting actin structure and function, vanadium can regulate many cellular processes of great physiological significance.
\end{abstract}

\section{Introduction}

The number of articles about "vanadium" in the last decade (2001-2010) doubled in comparison to the previous one (1991-2000), from 1147 to 2351, from which 420 are about "vanadium" and proteins", after a research at PubMed. Similarly, the number of papers selected with the word "actin" doubled in number, from 22827 to 39567 , when these last two decades are compared. However, studies about vanadium interaction with actin are seldom. In fact, very few studies concerning the interaction of vanadium with actin have been so far described. ${ }^{1-3}$ One of these first studies demonstrated that vanadate (vanadium(v)), upon binding to F-actin-ADP

\footnotetext{
${ }^{a}$ REQUIMTE/CQFB, Dpto Quimica, Faculdade de Ciências e Tecnologia, Universidade Nova de Lisboa, 2829-516 Caparica, Portugal

${ }^{b}$ CCMar, DCBB, Faculdade de Ciências e Tecnologia, Universidade do Algarve,8000-139Faro,Portugal.E-mail: maalves@ualg.pt; Fax: + 351289 800066; Tel: + 351289800905
}

subunits, increases the strength of actin-actin interactions, stabilizing the F-actin filament, ${ }^{2}$ the polymerized form of actin, for vanadate concentrations up to $2 \mathrm{mM}$, demonstrating a similar behaviour to phosphate, whereas higher vanadate concentrations destabilize F-actin. ${ }^{2}$ In a previous study, vanadate was also compared with phosphate on its ability to induce actin polymerization showing distinct effects. ${ }^{1}$ In another study, it was analysed that vanadyl, vanadium(Iv), interacts with the monomeric actin, G-actin, revealing the presence of one strong protein vanadium binding site, among others. ${ }^{3}$ More recently, it was demonstrated at our laboratory that also vanadate oligomers, such as tetrameric $\left(\mathrm{V}_{4}\right)$ and decameric vanadate (decavanadate, $\mathrm{V}_{10}$ ), prevent $\mathrm{G}$-actin polymerization, more potently than monomeric vanadate. ${ }^{4}$ In fact, it was shown that decavanadate, at concentrations as low as $68 \mu \mathrm{M}$, inhibits $50 \%$ of the extension of actin polymerization, whereas no effects were observed for monomeric vanadate $\left(\mathrm{V}_{1}\right)$ up to $2 \mathrm{mM}^{4}$ Moreover, it was also verified that monomeric actin (G-actin) stabilizes decavanadate species, by increasing its half-life time of 
decomposition, from 5 to 27 hours. ${ }^{4}$ Taken together, these results reveal a new protein target for decavanadate and a specific vanadium-protein interaction, confirmed later and described below.

Vanadium impact in biology, pharmacology and medicine is well known, mainly after the discovery that the "muscle inhibitor factor" present in ATP obtained from horse muscle and responsible for $\mathrm{Na}^{+}, \mathrm{K}^{+}$-ATPase inhibition was, in fact, vanadate. ${ }^{5}$ Vanadate acts as a transition state analogue for phosphoenzyme hydrolysis, blocking the enzyme catalysis of many enzymes, such as phosphatases and ATPases. In fact, vanadate is known as a potent inhibitor of protein tyrosine phosphatases (PTP), described as one of the main targets of vanadate as an insulin enhancement agent, promoting the increase of glucose uptake in several types of cells, among other effects that prevent diabetes. ${ }^{6}$ Additionally, the usage of vanadium as a tool to understand several biochemical processes is also well recognized, since it shows many biological activities. ${ }^{2,7}$

Actin is one of the most abundant proteins in cells, being involved in many cellular processes, such as cytoskeleton structure and dynamics in non-muscle cells, and muscle contraction in muscular cells. Cellular studies have shown that vanadium compounds induce changes in actin cytoskeleton, which are responsible for morphological and cell proliferation alterations. ${ }^{8,9}$ These effects are probably induced through the inhibition of protein tyrosine phosphatases, as referred above, or eventually through reactive oxygen species generation, hence it is well known that transition elements, such as vanadium, promote Fenton-like reactions. These actions could explain, at least in part, the increasing interest in vanadium for antitumor effects, ${ }^{10}$ as well as antidiabetic agents, this latter process being induced through an insulin dependent or insulin independent pathway, ${ }^{6,11,12}$ although the mechanisms of action are still to be clarified. It is believed that one of the main targets of vanadium in the cell will be actin, regulating or preventing many events. ${ }^{13,14}$ Therefore, it will be of extreme importance to clarify the mode of interaction and the effects of vanadium on actin structure and function. In the present report, a

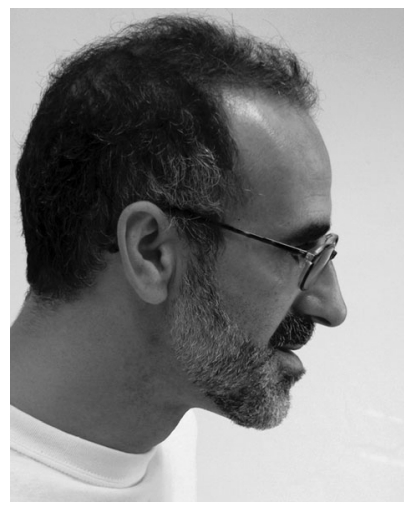

M. Aureliano
Dr Manuel Aureliano is an Associate Professor of Biochemistry at the Faculty of Sciences and Technology, University of Algarve, Faro, Portugal. He obtained his Biochemistry degree from Coimbra University, Portugal, where he also obtained his $\mathrm{MSc}$ and PhD degrees, working on decavanadate interactions with muscle myosin and sarcoplasmic reticulum calcium pump, respectively. He is regularly a peer reviewer (over 60) for numerous scientific journals (more than 20) and has served on the Editorial Boards of scientific journals. To date, he has supervised and/or co-supervised more than 80 post-doc, PhD, MSc and undergraduate students, and has published about 60 peer-reviewed journal articles, reviews and book chapters. combination of kinetic studies and ${ }^{51} \mathrm{~V}$ NMR, EPR, fluorescence and $\mathrm{UV} / \mathrm{V}$ is spectroscopy techniques was used to establish the interaction and/or effects of vanadyl, vanadate and decavanadate on actin structure and function.

\section{Decavanadate interaction with actin: cysteine oxidation and vanadyl formation}

As described above, it was revealed that decavanadate interacts with actin. It was verified, by ${ }^{51} \mathrm{~V}$ NMR, that decavanadate NMR signals broadened and decreased in intensity upon actin titration (Fig. 1), whereas no significant changes were observed for the other NMR vanadate oligomer signals. ${ }^{13}$ The broadening of $\mathrm{V}_{10}$ NMR signal, at physiological $\mathrm{pH}$ and for a $0.5 \mathrm{mM} \mathrm{V} \mathrm{V}_{10}$ concentration $(5 \mathrm{mM}$ total vanadium concentration), at $-516 \mathrm{ppm}$ (ascribed to decameric vanadate species), increased 2-fold, from 139 to about $299 \mathrm{~Hz}$, upon titration with G-actin up to $100 \mu \mathrm{M}$ (Fig. 1). Moreover, $\mathrm{V}_{10}$ signal decreases in intensity, with no changes observed in its chemical shift in the presence of the protein. In contrast, no effects were detected upon protein addition for the monomeric vanadate NMR signal, at $-560 \mathrm{ppm}$, with a half-width of $69 \mathrm{~Hz}$. At the same experimental conditions, but in the presence of $0.2 \mathrm{mM}$ ATP, the broadening effect was decreased from 2 to 1.4 (not shown), suggesting that ATP blocks decameric vanadate interactions with actin. Conversely, the presence of ATP induces the increase of the monomeric vanadate NMR signal upon actin titration, at $-560 \mathrm{ppm}$, from 70 to $83 \mathrm{~Hz}$ (1.2 fold), without significant changes in the chemical shift. Moreover, increasing the ionic strength, by $100 \mathrm{mM} \mathrm{KCl}$ addition, the decavanadate broadening effect was also reduced to 1.5 -fold (not shown), indicating an electrostatic interaction between the protein and decavanadate. Taken together, the presence of ATP promotes the broadening of monomeric NMR vanadate signal but it prevents the broadening of decavanadate signals, suggesting that this nucleotide promotes monomeric vanadate interaction with actin but on contrary blocks decavanadate interaction. ${ }^{15}$ The broadening of

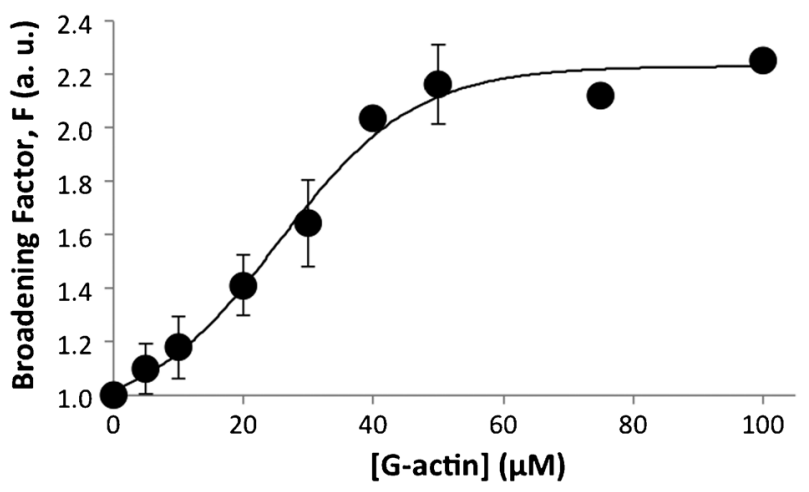

Fig. 1 Titration of decavanadate ( $5 \mathrm{mM}$ total vanadate) with $\mathrm{G}$-actin in a medium containing $2 \mathrm{mM}$ Tris ( $\mathrm{pH} 7.5), 0.2 \mathrm{mM} \mathrm{CaCl}_{2}$; data are plotted as means $\pm \mathrm{SD}$ and fitted with a Boltzmann sigmoidal function. Broadening factor, $F$, represents the quotient between the line width of the $\mathrm{V}_{10}$ signal, at $-516 \mathrm{ppm}$, in the absence of actin and upon protein addition. The results shown are the average of triplicate experiments. In certain experimental conditions the error bar is within the diameter of the symbol. 
$\mathrm{V}_{10}$ NMR signals, upon protein titration, was previously described for both myosin and $\mathrm{Ca}^{2+}$-ATPase, pointing out specific decavanadate interactions with these three proteins involved in the process of muscle contraction and its regulation. ${ }^{15,16}$ The broadening of monomeric vanadate, observed only in the presence of ATP, was also previously described, ${ }^{15}$ suggesting that ATP favours vanadate monomer interaction with the protein, probably through formation of an ATP analogue such as ATP.V or ADP.V. ${ }^{15,16}$ The broadening of NMR vanadate signals upon interaction with proteins has also been previously reported for several vanadate complexes. ${ }^{17-20}$

As observed by SDS-PAGE gel electrophoresis, the isolation of actin, according to Pardee and Spudich, ${ }^{21}$ produced a G-actin ( $42.3 \mathrm{kDa}$ ) with $99 \%$ purity (not shown), which allows concluding that the NMR observations described above are due to the interaction of the monomeric state of actin, G-actin, with decavanadate. On the other hand, the addition of actin in its polymerized form, F-actin, up to $50 \mu \mathrm{M}$, induces a much smaller broadening of $\mathrm{V}_{10}$ signals (1.5-fold), in comparison with G-actin, suggesting that decavanadate protein binding sites are encrusted upon actin polymerization. ${ }^{22}$

Once it was observed that the decavanadate NMR signal is affected upon actin interaction, it was asked: what will happen to the protein structure and function in the presence of several vanadium species, particularly with decavanadate? It is known that actin contains five cysteines that could react with vanadate, above all the so-called "fast cysteine" (Cys-374), more accessible to the solvent. Therefore, cysteine oxidation was analysed by $\mathrm{UV} / \mathrm{Vis}$ spectroscopy, and the titration of actin cysteines was performed with DTNB, as described previously ${ }^{13}$ using an extinction coefficient at $412 \mathrm{~nm}$ of $10900 \mathrm{M}^{-1} \mathrm{~cm}^{-1}$ for the colored product thionitrophenolate, ${ }^{13}$ after an exposure of 20 minutes to two different vanadate solutions, namely vanadate (also called metavanadate and containing $\mathrm{V}_{1}$ species) and decavanadate (containing $\mathrm{V}_{10}$ species). It was observed that only $\mathrm{V}_{10}$ solution, but not vanadate, was able to oxidize F-actin Cys374 and additionally one of the core cysteine residues (not shown), whereas for G-actin only the latter cysteine was affected (Fig. 2). In fact, in contrast to F-actin, the "fast cysteine" from G-actin remains in its reduced form, the oxidation of a core cysteine only being observed (Fig. 2). When ATP is present in the medium assay all five cysteine residues are still in their reduced form upon exposure to decavanadate for both G-actin and F-actin forms of the protein (not shown). Apparently, as described above using NMR spectroscopy, ATP protects the actin from the interaction with decavanadate, also preventing cysteine oxidation. ${ }^{14}$ Therefore, it was demonstrated that decavanadate interactions with actin are of particular interest hence it was observed that only $\mathrm{V}_{10}$ species are able to promote protein cysteine oxidation.

Reaction of vanadate with protein thiol groups from fructose-1,6-bisphosphate aldolase and from glyceraldehyde3-phosphate dehydrogenase, two enzymes involved in the glycolysis process, was previously referred. ${ }^{23,24}$ Moreover, it was also described that decavanadate is reduced by isocitrate dehydrogenase, ${ }^{25}$ an enzyme involved in the citric acid cycle, that is activated by adenosine nucleotide and calcium, but inhibited by ATP. The oxidation of actin cysteines upon decavanadate exposition leads to the question: does the oxidation of actin cysteines imply decavanadate reduction to vanadyl?

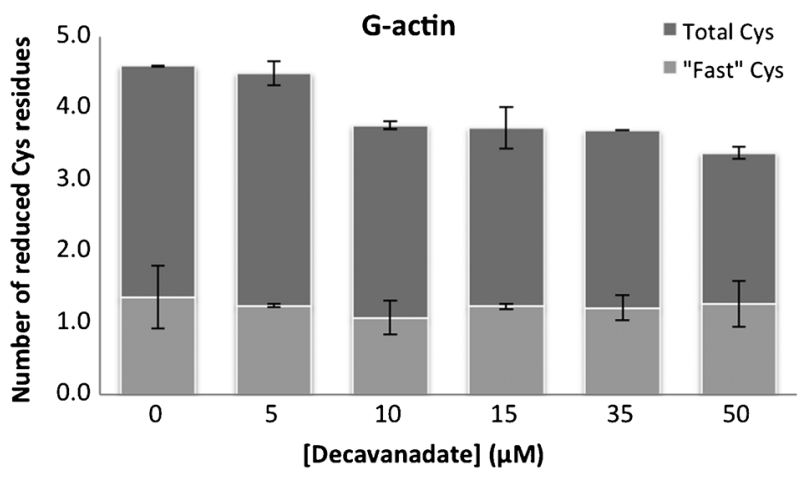

Fig. 2 G-actin cysteine redox state, after 20 minutes exposition with decavanadate. Titration of cysteines was performed with $0.1 \mathrm{mM}$ DTNB and $2 \mu \mathrm{M}$ actin in $2 \mathrm{mM}$ Tris (pH 7.5), and $0.2 \mathrm{mM} \mathrm{CaCl} 2$. The increase in absorbance at $412 \mathrm{~nm}$ was continuously recorded over $10 \mathrm{~min}$; to measure total cysteines the samples were treated afterwards with $1 \%$ SDS, and the absorbance was measured, over 15-30 min, until a steady value was reached. Titration with decavanadate produced a dose-dependent decrease of G-actin total cysteines, while Cys-374 remained in the reduced form. The results shown are the average of triplicate experiments.

In order to address this question, EPR studies were performed. In fact, decavanadate interaction with both G- and F-actin results in a concomitant vanadate reduction to vanadyl (V(IV)). ${ }^{13,14}$ Typical EPR vanadium(IV) signals can be detected upon decavanadate incubation with actin, whereas the presence of ATP in the medium, once again, prevents decavanadate reduction to vanadyl, as described above and reported previously. ${ }^{13,14}$ But once vanadate is reduced to vanadyl, does the reduced form of vanadium bind to actin?

In fact, as analysed by EPR spectroscopy, titration of vanadyl with $\mathrm{G}$-actin pointed out that vanadyl interacts with $\mathrm{G}$-actin. The extent of vanadyl binding to actin was measured from the $m_{\mathrm{i}}=-1 / 2$ perpendicular line of the EPR spectra (not shown) and the intensity of this line was plotted against vanadyl concentration (Fig. 3). It was calculated that vanadyl binds to actin with a $K_{\mathrm{d}}$ of $7.48 \pm 1.11 \mu \mathrm{M}^{-1}$ for G-actin and $43.05 \pm 5.34 \mu \mathrm{M}^{-1}$ for F-actin, with stoichiometry of approximately 1 and 4 vanadyl $\left(\mathrm{VO}^{2+}\right)$ cations bound per $\mathrm{G}$ - or F-actin molecule, respectively. ${ }^{13}$ Other studies performed with ferritin described a stoichiometry of 16 vanadyl cations to one protein molecule, ${ }^{26}$ this cation being recognized to bind to several proteins, at the same and higher orders of magnitude than the one described for actin. ${ }^{27-30}$ Similarly as it was described above for decavanadate, the presence of ATP in the assay medium prevents the interaction between vanadyl and actin, hence no EPR signals are detected. ${ }^{13}$

Recently reviewed decavanadate insights into biological systems have pointed out that this oligovanadate is either more or less efficient than the corresponding simple oxovanadates in targeting proteins, particularly at the nucleotide binding site. ${ }^{7,31}$ According to data presented in this paper, it is suggested that ATP prevents decavanadate interaction with actin, hence it blocks cysteine oxidation and vanadyl formation, among other effects described above. These observations suggest that a decavanadate protein binding domain would be eventually very close to or even at the nucleotide binding site, such as it happens for several proteins such as calcium 


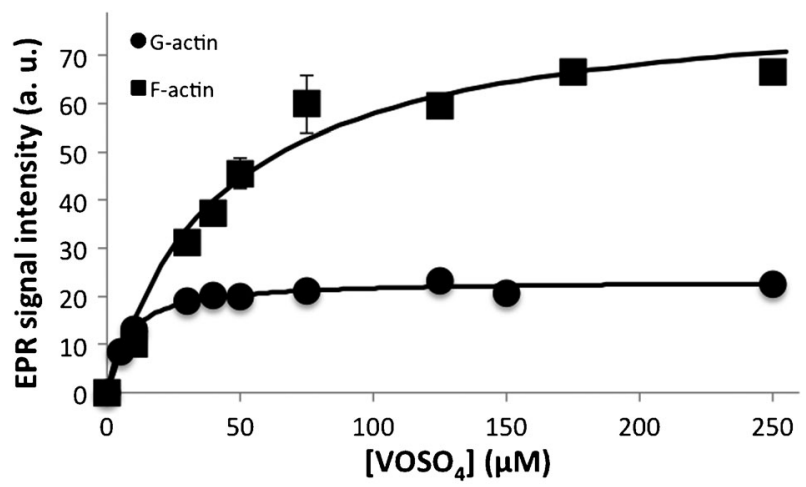

Fig. 3 Titration of both G-actin and F-actin with vanadyl. EPR intensities from the transition peak $m_{\mathrm{i}}=-1 / 2$ perpendicular line, of $50 \mu \mathrm{M}$ G-actin $(\bullet)$ or $30 \mu \mathrm{M}$ F-actin ( $\mathbf{a})$ versus concentrations of vanadyl sulfate. Medium containing $2 \mathrm{mM}$ Tris ( $\mathrm{pH} 7.5)$ and $0.2 \mathrm{mM}$ $\mathrm{CaCl}_{2}$. Data are plotted as means $\pm \mathrm{SD}$. The results shown are the average of triplicate experiments.

ATPase, adenylate kinase, myosin and ABC ATPases (ATP binding cassette ATPases), known to be inhibited by decavanadate. ${ }^{7,31}$ However, several studies must be performed to clarify the type and mode of decavanadate interaction with actin. Apparently, decavanadate seems to interact differently with the two forms of actin, the monomeric and the polymerized form. Therefore, it will be extremely interesting to understand the role of this oligovanadate ion in the several steps of the process of actin polymerization.

\section{Decavanadate and vanadyl effects on actin structure and function}

As described above, decavanadate revealed a very specific interaction with actin by inducing vanadate reduction and cysteine oxidation, with the concomitant binding of the cationic species to both monomeric and polymerized forms of actin. Previous studies referred to above described that vanadate could increase the strength of actin-actin interactions as phosphate does, but it could behave in a different mode to phosphate, during the process of actin polymerization, whereas vanadyl binds to actin. ${ }^{1-3}$ But do vanadate, vanadyl and decavanadate species affect, to the same extent, the process of actin polymerization and consequently many processes that would occur in cells? To address this question we analysed, by light scattering spectroscopy, the effects of all three vanadium species on the extension of G-actin polymerization into F-actin filaments (Fig. 4). Under exactly the same experimental conditions, the three vanadium species affected the actin polymerization in a very different way (Fig. 4). Although for both vanadyl and decavanadate the inhibition curve contains two behaviours, it was determined that the $\mathrm{IC}_{50}$ for the inhibition of polymerization reaction was lower for decavanadate by comparison with vanadyl $(68$ and $300 \mu \mathrm{M}$, respectively), whereas no effects were observed up to $2 \mathrm{mM}$ vanadate, as described previously for $8 \mu \mathrm{M}$ G-actin in the reaction medium. ${ }^{4,30}$ Therefore, for a decavanadate:actin ratio of 8.5 , decavanadate inhibits polymerization, whereas a vanadyl : actin ratio of 37.5 is needed to induce the same effect.

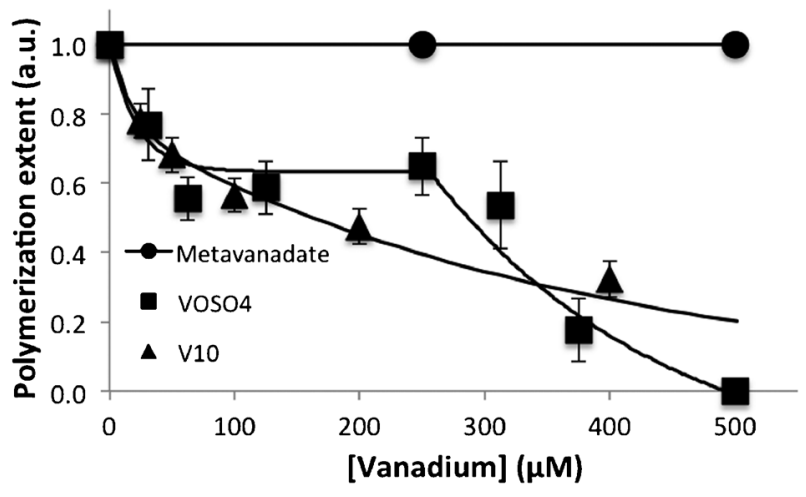

Fig. 4 Dependence of decavanadate $(\boldsymbol{\Delta})$, vanadyl $(\boldsymbol{\square})$ and vanadate (-) concentrations on the extent of G-actin polymerization relative to that in untreated G-actin (control) measured by the increase of light scattering intensity, at $\lambda_{\mathrm{ex}}=\lambda_{\mathrm{em}}=546 \mathrm{~nm}$. G-actin $(8 \mu \mathrm{M})$ was incubated with the different vanadium species for $20 \mathrm{~min}$, in a medium containing $2 \mathrm{mM}$ Tris- $\mathrm{HCl}(\mathrm{pH} 7.5)$ and $0.2 \mathrm{mM} \mathrm{CaCl}_{2}$, before the polymerization initiated by $100 \mathrm{mM} \mathrm{KCl}$ and $2 \mathrm{mM} \mathrm{MgCl}_{2}$, at $25^{\circ} \mathrm{C}$. Data are plotted as means $\pm \mathrm{SD}$. The results shown are the average of triplicate experiments.

In studies with other metals, it was described that a ratio of 9 for gadolinium : actin completely inhibited actin polymerization. ${ }^{32}$ Therefore, decavanadate is quite potent in preventing actin polymerization.

It was also verified, at the same F-actin concentrations described in the recent studies described above, that decavanadate and vanadyl species do not induce depolymerisation of the actin filaments to the same degree as they prevent actin polymerization. ${ }^{22}$ In fact, up to an $8 \mathrm{mM}$ vanadium total concentration, vanadate and decavanadate (meaning $0.8 \mathrm{mM} \mathrm{V_{10 }}$ ) induce F-actin depolymerisation by about $20 \%$ and $35 \%$, respectively, after two hours of incubation, whereas no effects were detected for vanadyl up to $0.5 \mathrm{mM} .^{22}$ Therefore, for the vanadium concentration of up to $0.5 \mathrm{mM}$ used in actin polymerization studies no significant effects on F-actin depolymerisation (less than 10\%) were detected for all the vanadium species. ${ }^{22}$

In order to further explore and compare the actin effects of vanadate, vanadyl and decavanadate, fluorescence spectroscopy studies were performed to address specific vanadium interactions with protein. It was verified that for vanadium concentrations as low as $200 \mu \mathrm{M}$, vanadyl induces a total quenching $(100 \%)$ of G-actin intrinsic fluorescence, whereas decavanadate increases its fluorescence up to 1.4 fold (Fig. 5). Regarding F-actin, at the same experimental conditions, no effects were detected on protein intrinsic fluorescence upon titration with vanadyl (not shown). The lack of quenching of F-actin intrinsic fluorescence by vanadyl suggests that the tryptophans present in the protein are protected from vanadium effects when the actin is present in its polymerized form. ${ }^{14,30}$ Eventually, actin conformational changes induced during actin polymerization prevent the vanadium quenching. However, for higher vanadyl concentrations from 200 to $500 \mu \mathrm{M}$, a maximum of $75 \%$ intrinsic protein quenching was observed, as described elsewhere. ${ }^{30}$ On the other hand, a full quenching of the intrinsic fluorescence of actin is observed for the monomeric state of the protein, G-actin, upon lower vanadyl 


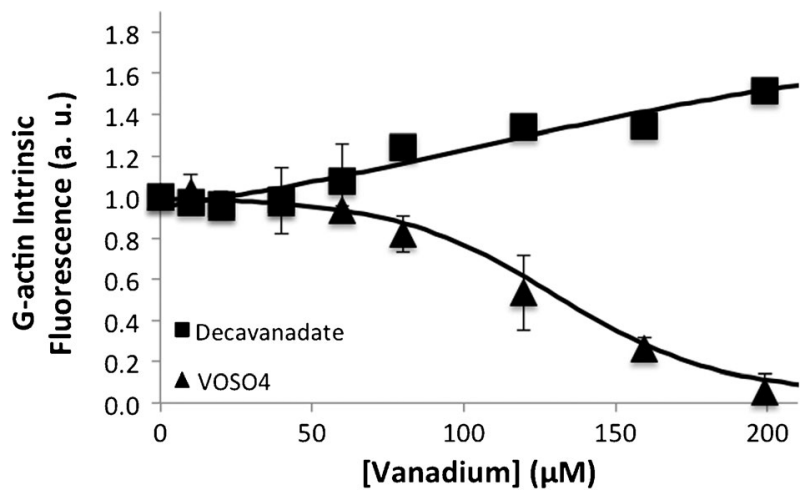

Fig. 5 Titration of G-actin intrinsic fluorescence with vanadyl ( $\mathbf{\Delta}$ and decavanadate $(\boldsymbol{\square})$. G-actin $(5 \mu \mathrm{M})$ was incubated for 20 minutes with vanadium species, in $2 \mathrm{mM}$ Tris- $\mathrm{HCl}(\mathrm{pH} 7.5), 0.2 \mathrm{mM} \mathrm{CaCl} \mathrm{Cl}_{2}$. The maximum of intrinsic fluorescence spectra $\left(\lambda_{\mathrm{ex}}=295 \mathrm{~nm}\right)$ was plotted against vanadium concentrations, considering 1.00 the value for native actin. The results shown are the average of, at least, triplicate measurements.

concentrations (0.2 mM) (Fig. 5). Usually, described vanadate protein quenching percentages are about 10 or $20 \%$, such as the ones verified for the quenching of myosin intrinsic fluorescence by decavanadate. ${ }^{14,33}$ The results obtained with vanadyl (total quenching) point out that, apparently, in actin, its four tryptophans are connected in a network in the tridimensional structure of the protein. On the other hand, the different residues contribute differently to the total fluorescence ${ }^{34}$ and they may be differently affected by the metal. Moreover, eventually, not all the several vanadyl binding sites contribute equally to protein intrinsic quenching. It is believed that further studies must be performed to clarify the vanadyl interaction with actin.

Regarding protein intrinsic fluorescence, care must be taken to evaluate the interaction of decavanadate or vanadate with proteins, due to inner filter effects. ${ }^{14,30}$ However, many biochemical studies using vanadium complexes and, particularly, decavanadate (with absorptions at 360 and $400 \mathrm{~nm}^{7,31}$ ), do not take into consideration those effects, which decreases the fluorescence measurements accuracy. For the maximum decavanadate concentration referred to in these studies, $20 \mu \mathrm{M}$, (meaning $0.2 \mathrm{mM}$ total vanadium), the absorbance values at excitation and emission wavelengths used in assays (295 and $340 \mathrm{~nm}$, respectively) attain to a sum of 0.841 O.D. $(0.583+$ $0.258)$, which is higher than the value obtained for monomeric vanadate $(0.475 ; 0.389+0.086)$ and for vanadyl $(0.085$; $0.064+0.021)$, for the same vanadium concentrations $(0.2 \mathrm{mM})$. Correction of the fluorescence intensities due to these inner filter effects is desirable, and can be done using proper equations, although its application is not clearly established, or does not state what conditions would be clearly appropriate, ${ }^{35}$ whereas some of them can be used even for values of absorption up to $2.7^{36}$ or as high as $5 .^{37}$

Besides the effects on intrinsic fluorescence, it was also observed that the actin hydrophobic surface, as determined using the ANSA probe, increases upon decavanadate exposure (2.6-fold), whereas vanadyl promotes its decrease by $15 \%$, suggesting that the changes caused by the former are clearly different from the ones induced by vanadyl, favouring a

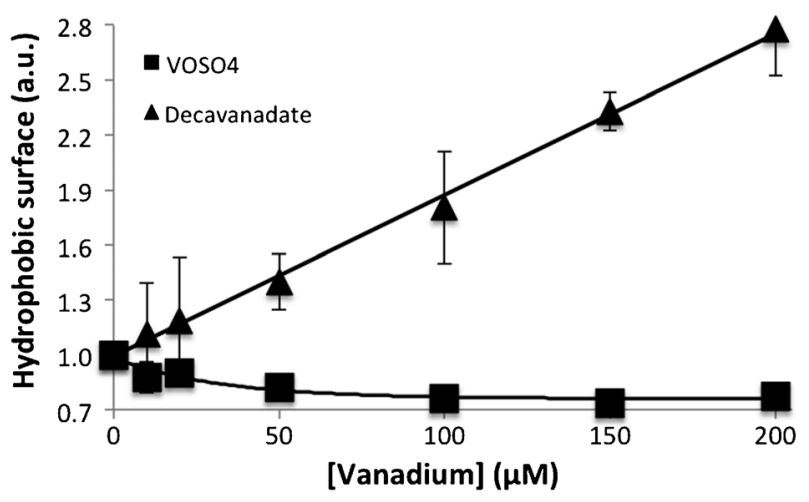

Fig. 6 Determination of G-actin surface hydrophobicity by ANSA binding. After treatment with different decavanadate $(\mathbf{\Lambda})$ and vanadyl (a) concentrations for $20 \mathrm{~min}$, in $2 \mathrm{mM}$ Tris $-\mathrm{HCl}(\mathrm{pH} 7.5), 0.2 \mathrm{mM}$ $\mathrm{CaCl}_{2}$, the change in fluorescence intensity associated with the binding of ANSA, to $9 \mu \mathrm{M}$ actin surface hydrophobic regions, was measured at $500 \mathrm{~nm}$ with $\lambda_{\mathrm{ex}}=370 \mathrm{~nm}$. Data are plotted as means $\pm \mathrm{SD}$. The results shown are the average of triplicate experiments.

protein hydrophobic environment (Fig. 6), as described recently. ${ }^{14,30}$ Therefore, decavanadate induced a less compact protein intermediate state than the one induced by vanadyl, wherein the hydrophobic interactions in the interior of the protein decreased, leading to an increased exposure of hydrophobic surface relative to the native structure. However, both decavanadate and vanadyl (up to $200 \mu \mathrm{M}$ total vanadium) increased the $\varepsilon$-ATP exchange rate $\left(k=6.5 \times 10^{-3} \mathrm{~s}^{-1}\right.$ and $4.47 \times 10^{-3} \mathrm{~s}^{-1}$, respectively, in comparison with the control: $k=3.0 \times 10^{-3} \mathrm{~s}^{-1}$ ), and both species decreased ATP exchange half-life time, denoting a more available cleft. ${ }^{14,30}$ Since it is known that the larger the value of the ATP exchange rate, the more available the cleft should be ${ }^{38}$ it can be concluded that vanadyl and decavanadate are clearly promoting structural alterations on actin ATP binding sites.

Actin conformational changes upon vanadyl exposure were also described by ${ }^{1} \mathrm{H}$ NMR. ${ }^{30}$ Besides the effects described with vanadyl, it was also observed, for the first time, and by ${ }^{1} \mathrm{H}$ NMR, that decavanadate induced several protein conformational changes (Fig. 7). G-actin exhibits a spectrum typical of a well-folded protein (Fig. 7, bottom spectrum), with the characteristic $\mathrm{N}$-terminal acetyl group resonance at $2.05 \mathrm{ppm}$; a second resonance can be observed upfield of this signal, which has been assigned to the methyl groups of two methionines. From the analysis of the ${ }^{1} \mathrm{H}$ NMR spectra, one can infer that $100 \mu \mathrm{M}$ decavanadate induces certain conformational changes on G-actin, that are readily observable by both chemical shift perturbations and decrease in the intensities of resonances located in the methyl group and aliphatic regions (1-3 ppm), as well as the aromatics and peptide-bond amide regions (7-8 ppm) (Fig. 7, middle spectrum). Moreover, the addition of $0.2 \mathrm{mM}$ of ATP to the medium results in a change in the actin structure, much less inferior than the one described above (not shown). ${ }^{22}$ This effect of ATP on preventing the conformational changes induced by decavanadate are in good agreement with the results described above regarding cysteine oxidation and vanadyl formation, among other effects, allowing us to confirm without any doubt that ATP prevents the interaction between the decameric vanadate species and actin. 


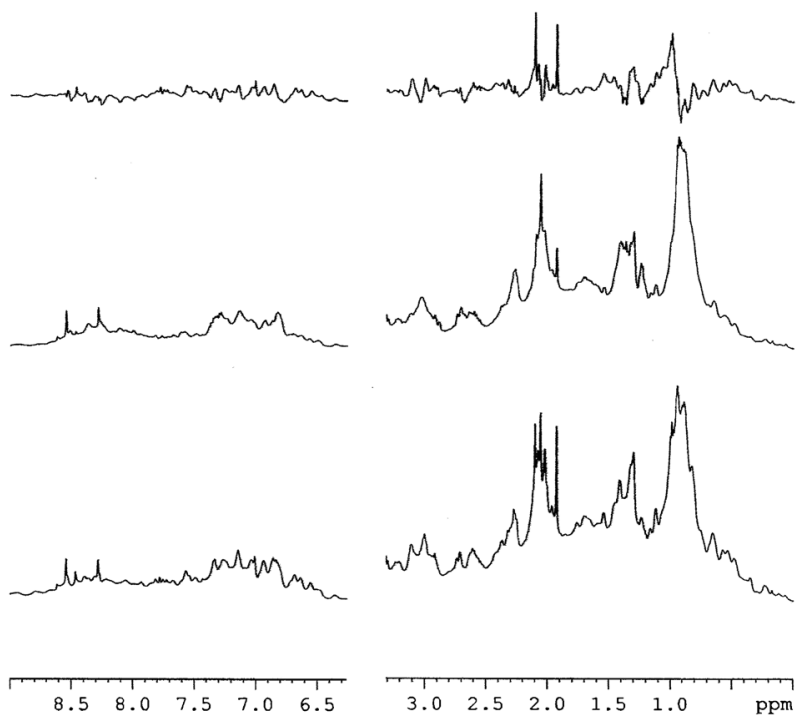

Fig. $7 \quad{ }^{1} \mathrm{H}$ NMR spectra of $63 \mu \mathrm{M}$ G-actin (bottom line) plus $0.1 \mathrm{mM}$ decavanadate (middle line) in the absence (A) and in the presence of $0.2 \mathrm{mM}$ ATP (B). The top spectra are the difference spectra. The spectra were obtained in the medium containing $2 \mathrm{mM}$ Tris- $\mathrm{HCl}$ ( $\mathrm{pH} 7.5$ ), $0.2 \mathrm{mM} \mathrm{CaCl}_{2}$. All spectra were acquired at a temperature of $298 \mathrm{~K}$ using a Bruker Avance III $600 \mathrm{MHz}$ spectrometer equipped with a TCI-Z cryoprobe and a temperature control unit. 256 free-induction decays were accumulated per spectrum, with an inter-scan delay of $1.5 \mathrm{~s}$.

The interactions of both vanadyl cation and vanadate anion with actin may be a key to understand their in vivo and in vitro effects in biology. ${ }^{39}$

As described above, the interaction of actin with decavanadate increases the stability of the anion. ${ }^{4}$ In studies with decavanadate, the half-life time should be always determined, normally between 5 to 12 hours $\left(25^{\circ} \mathrm{C}\right)$ and 3 hours $\left(37^{\circ} \mathrm{C}\right)$, depending on medium and temperature. ${ }^{7,31,40-43}$ Our studies were always performed during a reaction time much less than the decavanadate stability values, between 10 to 30 minutes, depending on the studies, in order to assure that the biological effects were mainly due to decavanadate., $4,13,14,16,31$ However, in the majority of the vanadium studies, the stability of the vanadate species or the vanadium complexes is not taken into account. If we do not take into account the stability of the vanadium species or complexes, we can only speculate about the observed effects. It was recently described that other vanadium species than the ones that are being studied can be observed under the experimental conditions. ${ }^{20}$ In fact, even for vanadium complexes, known to induce several insulin-like effects, it was verified that other species can be formed, with vanadium oxidation states other than the original one. ${ }^{20}$ Therefore, besides the factors known to describe the vanadium complex chemistry, namely, several oxidation states, similarity between phosphate and vanadate, the occurrence of several vanadate oligomers in solution and the formation of vanadium complexes with many molecules of biological interest, we may add the importance to address the stability of the vanadium complexes or species and to certify the presence of decameric vanadate species (responsible for the yellow colour of vanadate solutions), before to attempt to attribute a certain biological activity or effect to a vanadium complex or species.

\section{Concluding remarks}

A combination of ${ }^{51} \mathrm{~V}$ NMR, EPR, UV/Vis and fluorescence spectroscopy techniques was used to determine the effects of several vanadium species, namely vanadyl, vanadate and decavanadate on G-actin and F-actin structure and function. The studies reveal the presence of a vanadyl G-actin high affinity binding sites, with a 1:1 actin: vanadium(Iv) stoichiometry. Also, a specific decavanadate interaction with actin was observed, leading to cysteine oxidation and vanadyl formation. Both decavanadate and vanadyl interactions with actin were prevented by ATP. Putting it all together, it is proposed that the biological effects of vanadium, whose major biological role is still to be clarified, may be explained, at least in part, by its capacity to interact with actin and to affect several biological processes where actin may be involved. It is concluded in this paper that: (i) decavanadate and vanadyl inhibit actin polymerization, at $\mu \mathrm{M}$ concentrations; (ii) only decavanadate interaction with actin induces cysteine oxidation and vanadyl formation, these effects being prevented by ATP; (iii) decavanadate and vanadyl induce actin conformational changes affecting the protein ATP binding site; (iv) actin has high affinity binding sites for vanadyl. It is suggested that actin, a protein involved in many cellular processes, is a plausible protein target for decavanadate and, above all, for vanadyl. By affecting actin structure and function vanadium can regulate several cellular processes of great physiological significance. It is believed that spontaneous interactions of both vanadyl cation and vanadate anion with actin may be a key to understand both in vivo and in vitro effects in biological processes involving muscle and non-muscle actin.

In the present decade, we expect that important questions will be answered, for instance: (i) will we be able to characterize the first X-ray structures of vanadyl-actin and decavanadateactin complexes? (ii) Will we understand the role of vanadyl, decavanadate and vanadate in the several steps of the process of actin polymerization/depolymerisation? (iii) Will we be able to understand the contribution of vanadium interaction with actin to the effects of vanadium as insulin enhancement and as anticancer agents, among other effects? These questions and others will require continuous development of new approaches to explore the vanadium effects in these complex systems.

\section{Abbreviations \\ ANSA 8-anilino-1-naphthalene sulfonic acid \\ DTNB 5,5'-dithio-bis(2-nitrobenzoic acid) \\ G-actin monomeric actin \\ F-actin filamentous polymerized actin}

\section{Acknowledgements}

This work was supported by CCMAR funding. S. Ramos would like to thank the Portuguese "Fundação para a Ciência e Tecnologia" (FCT) for the PhD grant SFRH/BD/29712/ 2006.

\section{References}

1 S. C. El-Saleh and P. Johnson, Int. J. Biol. Macromol., 1982, 4, 430. 2 C. Combeau and M.-F. Carlier, J. Biol. Chem., 1988, 263, 17429. 
3 F. An, B. Y. Zhang, B. W. Chen and K. Wang, Chem. J. Chin. Univ., 1996, 17, 667.

4 S. Ramos, M. Manuel, T. Tiago, R. O. Duarte, J. Martins, C. Gutiérrez-Merino, J. J. G. Moura and M. Aureliano, J. Inorg. Biochem., 2006, 100, 1734.

5 L. C. Cantley Jr., L. Josephson, R. Warner, M. Yanagisawa, C. Lechene and G. Guidotti, J. Biol. Chem., 1977, 252, 7421.

6 E. Tsiani, E. Bogdanovic, A. Sorisky, L. Nagy and I. G. Fantus, Diabetes, 1998, 47, 1676.

7 M. Aureliano and D. C. Crans, J. Inorg. Biochem., 2009, 103, 536.

8 X.-G. Yang, X.-D. Yang, L. Yuan, K. Wang and D. C. Crans, Pharm. Res., 2004, 21, 1026.

9 J. Rivadeneira, D. A. Barrio, G. Arrambide, D. Gambino, L. Bruzzone and S. B. Etcheverry, J. Inorg. Biochem., 2009, 103, 633.

10 A. M. Evangelou, Crit. Rev. Oncol. Hematol., 2002, 42, 249.

11 F. Yraola, S. Garcia-Vicente, L. Marti, F. Albericio, A. Zorzano and M. Royo, Chem. Biol. Drug Des., 2007, 69, 423.

12 J. Li, G. Elberg, N. Sekar, Z. B. He and Y. Shechter, Endocrinology, 1997, 138, 2274.

13 S. Ramos, R. O. Duarte, J. J. G. Moura and M. Aureliano, Dalton Trans., 2009, 7985.

14 S. Ramos, J. J. G. Moura and M. Aureliano, J. Inorg. Biochem., 2010, 104, 1234.

15 M. Aureliano and V. M. C. Madeira, Biochim. Biophys. Acta, Mol. Cell Res., 1994, 1221, 259.

16 T. Tiago, M. Aureliano and C. Gutierrez-Merino, Biochemistry, 2004, 43, 5551.

17 A. Butler, M. J. Danzitz and H. J. Eckert, J. Am. Chem. Soc., 1987, 109, 1864.

18 P. J. Stankiewicz, M. J. Gresser, A. S. Tracey and L. F. Hass, Biochemistry, 1987, 26, 1264.

19 L. Wittenkeller, W. Lin, C. Diven, A. Ciaccia, F. Wang and D. Mota de Freitas, Inorg. Chem., 2001, 40, 1654.

20 M. Aureliano, F. Henao, T. Tiago, R. O. Duarte, J. J. G. Moura, B. Baruah and D. C. Crans, Inorg. Chem., 2008, 47, 5677.

21 J. D. Pardee and J. A. Spudich, Methods Enzymol., 1982, 85, 164.

22 S. Ramos, PhD Thesis, New University of Lisbon, 2011.

23 I. Dalle-Donne, R. Rossi, D. Giustarini, N. Gagliano, L. Lusini, A. Milzani, P. Di Simplicio and R. Colombo, Free Radical Biol. Med., 2001, 31, 1075.
24 D. C. Crans, K. Sudhakar and T. J. Zamborelli, Biochemistry, 1992, 31, 6812.

25 J. E. Benabe, L. A. Echegoyen, B. Pastrana and M. MartınezMaldonado, J. Biol. Chem., 1987, 262, 9555.

26 N. D. Chasteen and E. C. Theil, J. Biol. Chem., 1982, 257, 7672.

27 N. D. Chasteen, in Structure and Bonding, ed. M. J. Clarke, J. B. Goodenough, J. A. Ibers, C. K. Jørgensen, D. M. P. Mingos, J. B. Neilands, G. A. Palmer, D. Reinen, P. J. Sadler, R. Weiss and R. J. P. Williams, Springer-Verlag, New York, 1983, pp. 105-138.

28 L. WanHua, L. HuiXue, Z. LiJun, Y. XiaoDa and K. Wang, Chin. Sci. Bull., 2007, 52, 2775.

29 E. G. Ferrer, A. Bosch, O. Yantorno and E. J. Baran, Bioorg. Med. Chem., 2008, 16, 3878.

30 S. Ramos, R. M. Almeida, J. J. G. Moura and M. Aureliano, J. Inorg. Biochem., 2011, 105, 777.

31 M. Aureliano, Dalton Trans., 2009, 9093.

32 C. G. dos Remédios and J. A. Barden, Biochem. Biophys. Res. Commun., 1977, 77, 1339.

33 T. Tiago, M. Aureliano and C. Gutiérrez-Merino, J. Fluoresc., $2002,12,87$.

34 K. K. Turoverov and I. M. Kuznetsova, J. Fluoresc., 2003, 13, 41.

35 J. R. Lackowicz, Principles of Fluorescence Spectroscopy, Plenum Press, New York, 1983, p. 56.

36 M. Kubista, R. Sloback, S. Eriksson and B. Albinsson, Analyst, 1994, 119, 417.

37 B. Birdsall, R. W. King, M. R. Wheeler, C. A. Lewis Jr, S. R. Goode, R. B. Dunlap and G. C. Roberts, Anal. Biochem., 1983, 132, 353.

38 I. Dalle-Donne, R. Rossi, D. Giustarini, N. Gagliano, P. Di Simplicio, R. Colombo and A. Milzani, Free Radical Biol. Med., 2002, 32, 927.

39 D. C. Crans, R. L. Bunch and L. A. Theisen, J. Am. Chem. Soc., 1989, 111, 7597.

40 M. Aureliano, N. Joaquim, A. Sousa, H. Martins and J. M. Coucelo, J. Inorg. Biochem., 2002, 90, 159.

41 R. Gândara, S. S. Soares, H. Martins and M. Aureliano, J. Inorg. Biochem., 2005, 99, 2355.

42 S. S. Soares, H. Martins, R. O. Duarte, J. J. G. Moura, J. Coucelo, C. Gutiérrez-Merino and M. Aureliano, J. Inorg. Biochem., 2007, 101, 80.

43 S. S. Soares, C. Gutiérrez-Merino and M. Aureliano, Aquat. Toxicol., 2007, 83, 1 . 